\title{
LEVANTAMENTO FITOSSOCIOLÓGICO DA COMUNIDADE INFESTANTE EM GRAMADO SEMEADO COM Paspalum notatum FLÜGGE ${ }^{1}$
}

\author{
Cleber Daniel de Goes Maciel², Jessica Tiemi Hama², João Igor de Souza²
}

\section{ABSTRACT \\ PHYTOSOCIOLOGICAL ANALYSIS OF WEED \\ COMMUNITY IN Paspalum notatum FLÜGGE TURFGRASS}

A phytosociological analysis was carried out in Ourinhos, São Paulo State, Brazil, in order to evaluate the weed community behavior prevalent in $P$. notatum Flügge turfgrass, recently installed through sowing. Four evaluations were accomplished, between March and May (2007), after turfgrass and weeds emergence. Through the analysis, 23 weed species were identified, distributed in nine families, pointing out the number of species per family for Poaceae and Malvaceae. The Sida glaziovii (Malvaceae), Cyperus flavus (Cyperaceae), Cyperus rotundus (Cyperaceae), Chamaecrista rotundifolia (Leguminosae), and Cynodon dactylon (Poaceae) species showed the highest relative frequencies; S. glaziovii, Chamaesyce hyrta (Euphorbiaceae), C. flavus, Crotalaria lanceolata (Leguminosae), Sida cordifolia (Malvaceae), and Sida spinosa (Malvaceae) the highest abundance level; and $S$. glaziovii $>$ C. flavus $>$ C. rotundus $>$ C. rotundifolia $>$ C. dactylon the highest importance value index (IVI).

KEY-WORDS: Weeds; Bahiagrass; phytosociology.

A espécie Paspalum notatum Flügge é a grama mais disseminada, nas mais diferentes regiões do Brasil. Os gramados de grama-matogrosso ou gramabahia, entre outras denominações, são comumente utilizados com diferentes propósitos e em diversos locais, tais como áreas residenciais, industriais, urbanas e rodovias.

As plantas daninhas podem acarretar baixa produtividade ao gramado e perda de qualidade estética, quando há finalidade ornamental, pois concorrem por água, luz, nutriente e espaço, sendo, até mesmo, capazes de dizimá-lo completamente (Modesto Júnior \& Mascarenhas 2001, Freitas et al. 2003). Entretanto, informações sobre a frequência da comunidade infestante prevalente em gramados, nas diferentes regiões do Brasil, são escassas, resul-

\section{RESUMO}

Com o objetivo de avaliar o comportamento da comunidade infestante prevalecente em gramado $P$. notatum Flügge, recém-instalado através de semeadura, um levantamento fitossociológico foi realizado no Município de Ourinhos (SP). Quatro avaliações foram realizadas, entre março e maio de 2007, logo após o início da emergência do gramado e das plantas daninhas. No levantamento, foram identificadas 23 espécies de plantas daninhas, distribuídas em nove famílias, com destaque para o número de espécies por família para Poaceae e Malvaceae. As espécies Sida glaziovii (Malvaceae), Cyperus flavus (Cyperaceae), Cyperus rotundus (Cyperaceae), Chamaecrista rotundifolia (Leguminosae) e Cynodon dactylon (Poaceae) registraram as maiores frequências relativas; S. glaziovii, Chamaesyce hyrta (Euphorbiaceae), C. flavus, Crotalaria lanceolata (Leguminosae), Sida cordifolia (Malvaceae) e Sida spinosa (Malvaceae) a maior abundância; e S. glaziovii > C. flavus $>$ C. rotundus $>C$. rotundifolia $>C$. dactylon o maior índice de valor de importância (IVI).

PALAVRAS-CHAVE: Plantas daninhas; grama batatais; fitossociologia.

tando na importação desenfreada das mesmas e/ou generalização de soluções e recomendações sem base científica (Barbosa et al. 1999).

Dessa forma, este trabalho teve como objetivo identificar e quantificar as plantas daninhas de um gramado de $P$. notatum Flügge de campo de futebol, recém-instalado através de semeadura, no município de Ourinhos (SP).

O levantamento fitossociológico da comunidade infestante foi desenvolvido entre março e maio de 2007, na forma de quatro avaliações, com intervalo de 15 dias, realizadas após o início da emergência do gramado e das plantas daninhas. A semeadura do gramado foi realizada a lanço, em área de 40 × 60 m² (16/01/2007), através da distribuição em solo classificado como Nitossolo Vermelho Estru- 
turado, com textura argilosa, utilizando-se $50 \mathrm{~kg}$ de sementes, com $90 \%$ de pureza, $60 \%$ de germinação e valor cultural de $54 \%$.

As repetições foram representadas por 40 pontos amostrais, definidos, aleatoriamente, a cada 10 metros lineares, com auxílio de quadrados vazados de $0,25 \mathrm{~m}^{2}$, de forma semelhante às metodologias descritas por Maciel et al. (2008).

Em função dos dados obtidos, foram determinados os seguintes parâmetros: Frequência (ocorrência das espécies em cada quadrado); Densidade (quantidade de indivíduos de uma mesma espécie, em cada quadrado); Abundância (concentração das espécies nos diferentes pontos da área total $\left.-50,0 \mathrm{~m}^{2}\right)$; e Índice de Valor de Importância (espécies que mais ocorrem em cada área), de forma semelhante ao trabalho desenvolvido por Brandão et al. (1998):

Frequência Relativa $($ Frr) $=$ Frequência da espécie x 100 Frequência total de todas as espécies

Densidade Relativa $($ Der $)=\underline{\text { Densidade da espécie x } 100}$ Densidade total de todas as espécies

Abundância Relativa $(\mathrm{Abr})=\underline{\text { Abundância da espécie x } 100}$ Abundância total de todas as espécies

Índice de Valor de Importância (IVI) $=$ Frr + Der + Abr

O levantamento fitossociológico das plantas daninhas totalizou 662 indivíduos, representados por 23 espécies, distribuídas em nove diferentes famílias. As famílias Malvaceae (5) e Poaceae (5) foram as que registraram os maiores números de espécies no levantamento, seguidas pelas famílias Fabaceae (3) e Euphorbiaceae (3) (Figura 1).

Em ordem decrescente, as espécies que registraram as maiores frequências relativas (Frr) foram Sida glaziovii (Malvaceae), Cyperus flavus (Cyperaceae), Cyperus rotundus (Cyperaceae), Chamaecrista rotundifolia (Fabaceae-Caesalpinioideae) e Cynodon dactylon (Poaceae), respectivamente representadas por níveis de $23,4 \% ; 12,7 \% ; 8,2 \% ; 8,2 \%$; e $7,6 \%$. Com relação à densidade (Der), destacou-se a espécie Sida glaziovii $\left(13,20\right.$ plantas $\left.\mathrm{m}^{-2}\right)$, pertencente à família Malvaceae (Tabela 1).

Para a abundância (Tabela 1), parâmetro importante que revela as espécies que apareceram em reboleiras ou de forma dominante, destacaram-se, no levantamento, as espécies S. glaziovii (Malvaceae), Chamaesyce hyrta (Euphorbiaceae), C. flavus (Cyperaceae), Crotalaria lanceolata (FabaceaeFaboidae), Sida cordifolia (Malvaceae) e Sida spinosa (Malvaceae). Neste sentido, a informação sobre a abundância pode indicar a melhor estratégia para o controle das referidas espécies, uma vez que, segundo Christoffoleti \& Aranda (2001) e Freitas et al. (2003), no Brasil, estudos sobre o manejo de plantas infestantes em gramados e seletividade de herbicidas ainda são escassos.

Com relação ao valor de importância (IVI), as cinco principais espécies presentes no levantamento, em ordem decrescente de importância, foram S. glaziovii $>$ C. flavus $>C$. rotundus $>$

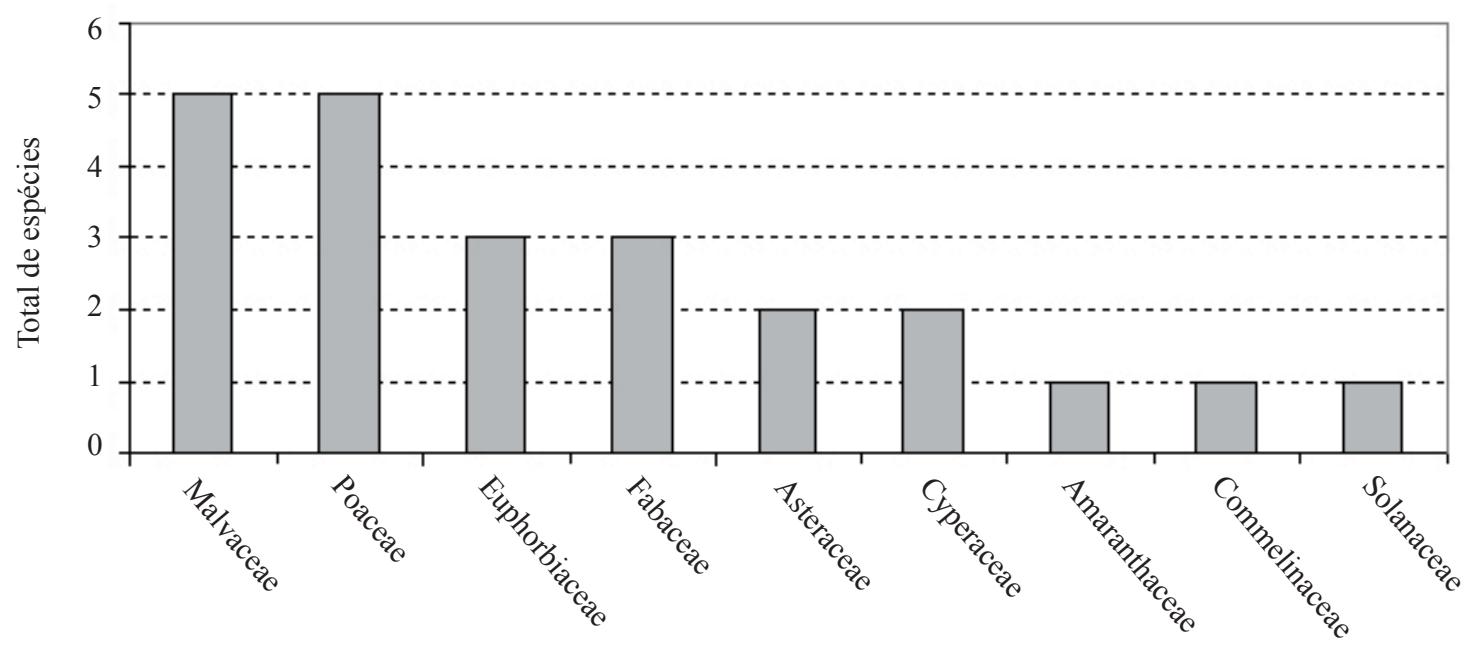

Figura 1. Número total de espécies de plantas daninhas, organizadas por família, identificadas em levantamento fitossociológico de gramado semeado com $P$. notatum Flügge. 
Tabela 1. Organização das espécies de plantas daninhas identificadas em levantamento fitossociológico de gramado semeado com P. notatum Flügge.

\begin{tabular}{cccccccc}
\hline Espécie & Família & $\begin{array}{c}\mathrm{N}^{\circ} \mathrm{de} \\
\text { quadros }\end{array}$ & $\begin{array}{c}\mathrm{N}^{\circ} \mathrm{de} \\
\text { indivíduos }\end{array}$ & Frr & Der & Abr & IVI \\
\hline Sida glaziovii & Malvaceae & 37 & 330 & 23,4 & 49,9 & 15,0 & 88,3 \\
Cyperus flavus & Cyperaceae & 20 & 97 & 12,6 & 14,7 & 8,2 & 35,5 \\
Cyperus rotundus & Cyperaceae & 13 & 40 & 8,2 & 6,0 & 5,2 & 19,5 \\
Chamaecrista rotundifolia & Fabaceae-Caesalpinioideae & 13 & 31 & 8,2 & 4,7 & 4,0 & 16,9 \\
Cynodon dactylon & Poaceae & 12 & 27 & 7,6 & 4,1 & 3,8 & 15,5 \\
Sida cordifolia & Malvaceae & 6 & 23 & 3,8 & 3,5 & 6,5 & 13,7 \\
Sida rhombifolia & Malvaceae & 8 & 21 & 5,1 & 3,2 & 4,4 & 12,7 \\
Brachiaria plantaginea & Poaceae & 11 & 19 & 7,0 & 2,9 & 2,9 & 12,7 \\
Brachiaria decumbens & Poaceae & 9 & 17 & 5,7 & 2,6 & 3,2 & 11,4 \\
Sida spinosa & Malvaceae & 3 & 11 & 1,9 & 1,7 & 6,2 & 9,7 \\
Crotalaria lanceolata & Fabaceae-Faboidae & 2 & 8 & 1,3 & 1,2 & 6,7 & 9,2 \\
Chamaesyce prostata & Euphorbiaceae & 3 & 7 & 1,9 & 1,1 & 3,9 & 6,9 \\
Mimos pudica & Fabaceae-Mimosoidae & 5 & 7 & 3,2 & 1,1 & 2,4 & 6,6 \\
Chamaesyce hyrta & Euphorbiaceae & 1 & 5 & 0,6 & 0,8 & 8,4 & 9,8 \\
Amaranthus deflexus & Amaranthaceae & 5 & 5 & 3,2 & 0,8 & 1,7 & 5,6 \\
Chamaesyce hyssopifolia & Euphorbiaceae & 2 & 4 & 1,3 & 0,6 & 3,4 & 5,2 \\
Herissantia crispa & Malvaceae & 2 & 3 & 1,3 & 0,5 & 2,5 & 4,2 \\
Physalis angulata & Solanaceae & 1 & 2 & 0,6 & 0,3 & 3,4 & 4,3 \\
Acanthospermum hispidum & Asteraceae & 1 & 1 & 0,6 & 0,2 & 1,7 & 2,5 \\
Cenchrus ciliaris & Poaceae & 1 & 1 & 0,6 & 0,2 & 1,7 & 2,5 \\
Commelina benghalensis & Commelinaceae & 1 & 1 & 0,6 & 0,2 & 1,7 & 2,5 \\
Digitaria horizontalis & Poaceae & 1 & 1 & 0,6 & 0,2 & 1,7 & 2,5 \\
Emilia sonchifolia & Asteraceae & 1 & 1 & 0,6 & 0,2 & 1,7 & 2,5 \\
\hline & & 158 & 662 & 100 & 100 & 100 & 300 \\
\hline
\end{tabular}

$\mathrm{N}^{o}$ de indivíduos = número total de indivíduos por espécie; $\mathrm{N}^{\circ}$ de quadros = número de quadros com a espécie; Frr = frequência relativa; Der = densidade relativa; Abr = abundância relativa; IVI = índice de valor de importância.

C. rotundifolia $>$ Cynodon dactylon (Tabela 1). Maciel et al. (2008) também relataram espécies do gênero Cyperaceae com alto valor de importância, em levantamento florístico em gramado de $P$. notatum Flügge já instalado.

Pode-se concluir que as famílias Malvaceae e Poaceae, seguidas das famílias Fabaceae e Euphorbiaceae, registraram os maiores números de espécies de plantas daninhas no levantamento fitossociológico, apesar de as famílias Cyperaceas C. flavus, C. rotundus e C. rotundifolia também terem se destacado como espécies mais importantes.

\section{REFERÊNCIAS}

BARBOSA, J. G. et al. Plantio de gramados de Paspalum notatum Flügge "batatais": tipos de mudas e doses de fertilizantes. Revista Brasileira de Horticultura Ornamental, Campinas, v. 3, n. 2, p. 50-54, 1999.
BRANDÃO, M.; BRANDÃO, H.; LACA-BUENDIA, J. P. A mata ciliar do Rio Sapucaí, município de Santa Rita do Sapucaí-MG: fitossociologia. Daphne, Belo Horizonte, v. 8, n. 4 , p. 36-48, 1998.

CHRISTOFFOLETI, P. J.; ARANDA, A. N. Seletividade de herbicidas a cinco tipos de gramas. Planta Daninha, Viçosa, v. 19, n. 2, p. 273-278, 2001.

FREITAS, F. C. L. et al. Eficiência do triclopyr no controle de plantas daninhas em gramado (Paspalum notatum). Planta Daninha, Viçosa, v. 21, n. 1, p. 159-164, 2003.

MACIEL, C. D. G. et al. Comportamento florístico da comunidade infestante em gramados de Paspalum notatum Flügge no Município de Assis, SP. Planta Daninha, Viçosa, v. 26, n. 1, p. 54-64, 2008.

MODESTO JÚNIOR, M. S.; MASCARENHAS, R. E. B. Levantamento da infestação de plantas daninhas associadas a uma pastagem cultivada de baixa produtividade no nordeste paraense. Planta Daninha, Viçosa, v. 19, n. 1, p. 11-21, 2001. 